\title{
Guest Editorial: Special Issue on Embedded Machine Learning
}

\author{
Sek Chai ${ }^{1} \cdot$ Senem Velipasalar ${ }^{2}$ \\ Received: 17 February 2021 / Revised: 17 February 2021 / Accepted: 17 February 2021 / Published online: 4 March 2021 \\ (C) The Author(s), under exclusive licence to Springer Science+Business Media, LLC part of Springer Nature 2021
}

The aim of this special issue is to highlight the state of the art in terms of research focused on machine learning systems. Machine learning, and deep neural networks in particular, have received a lot of attention recently, especially after achieving very good performance in detection and classification tasks. Since deep neural network-based approaches are computationally intensive, researchers have been working on hardware, software, and algorithmic approaches to develop resource-efficient platforms for machine learning.

This issue consists of five papers that are briefly discussed as follows:

In "MAHASIM: Machine-Learning Hardware Acceleration Using a Software-Defined Intelligent Memory System (Bahar Asgari, et.al.)", the authors present a processor hardware architecture based on programmable memory system to address challenges energy and performance of embedded machine learning.

In "New Algorithms for the Estimation of TwoDimensional Cyclic Spectral Information Based on Tensor Equations (Mehdi Chehel Amiran, et.al.)", the authors present an algorithmic approach to machine learning that leverages the temporal periodicity of signals, to improve the overall classification of multi-dimensional analysis.

Sek Chai

sek@latentai.com

Senem Velipasalar

svelipas@syr.edu

1 Latent AI, 30 Vreeland Dr, Suite 1, Princeton, NJ 08558, USA

2 Syracuse University, 111 College Pl., Syracuse, NY 13244, USA
In "On-device Partial Learning Technique of Convolutional Neural Network for New Classes (Sanggil Kang, et. al.)", the authors present a method for on-device learning to improve overall algorithmic accuracy post initial training.

In "Regular Contribution Multi-level Cell Progressive Differentiable Architecture Search to Improve Image Classification Accuracy (YuGang Shan, et. al.)", the authors present a training method using network architecture search to select efficient neural network configurations.

In "An Efficient Human Identification through Iris Recognition System (mamta Garg, et. al.)", the authors present a biometric application using machine learning to improve pattern recognition in iris features.

These five contributions encompass a wide range of topics, including data collection, machine learning training, system implementation, and benchmarking. Collectively, they highlight the breadth of machine learning systems research, and the diverse range of issues currently investigated in this field.

Publisher's Note Springer Nature remains neutral with regard to jurisdictional claims in published maps and institutional affiliations. 